\title{
View-Dependent Information Theory Quality Measures for Pixel Sampling and Scene Discretization in Flatland
}

\author{
Jaume Rigau \\ Institut d'Informàtica i Aplicacions \\ Universitat de Girona \\ jaume.rigau@udg.es \\ Philippe Bekaert \\ Max-Planck-Institut fuer Informatik \\ philippe@mpi-sb.mpg.de
}

\author{
Miquel Feixas \\ Institut d'Informàtica i Aplicacions \\ Universitat de Girona \\ miquel.feixas@udg.es \\ Mateu Sbert \\ Institut d'Informàtica i Aplicacions \\ Universitat de Girona \\ mateu.sbert@udg.es
}

\begin{abstract}
In this paper we present view-dependent information theory quality measures for pixel sampling and scene discretization in flatland. The measures are based on a definition for the mutual information of a line, and have a purely geometrical basis. Several algorithms exploiting them are presented and compare well with an existing one based on depth differences.
\end{abstract}

\section{Introduction}

Recently from the image based rendering and interactive rendering fields the need to measure the pixel quality or the triangle quality out of a mesh has appeared.

In [6], several images were blended to get the new one corresponding to the virtual camera. The color of a pixel of each image to be blended was weighted with a quality factor, which included the cosines of the angle between the normal to the surface and the viewing direction.

Darsa [3] uses an adaptive sampling strategy based on a priority schema that takes two factors into consideration, the difference in color between adjacent samples and the size of cells. The Gouraud-shaded constant color cells are triangles constructed using a Voronoi diagram or Delaunay triangulation, where each Voronoi edge corresponds to a pair of adjacent samples. In [4] they introduce into the sampling strategy the inverse of the depth, this is, they say "the mesh should approximate the object edges, and its sampling density should be inversely proportional to the depth".

In [11] a spherical Delaunay triangular mesh was created according to a priority schema. The priority was an estimate on how well a Gouraud-shaded triangle approximates the corresponding portion of the sample environment relative to the current view. The priority value was computed weighting two measures: a contrast measure for colour differences and a depth value based on depth differences relative to the current view. This scheme was used to select the next pixels to sample.

We will try to give here a unifying approach to deal with both pixel quality and priority schema sampling, based on information theory. Our approach is based on the definition for the mutual information of a line in flatland. For the moment being our approach covers only the geometry measure, but we are working on an extension to colour. We will show how the mutual information based measures are a natural representation for the quality of a pixel or a mesh, and that previous measures can be considered as approximating steps.

The organization of this paper is as follows: In section 2 we present our previous work about information theory measures in a 2D scene. In section 3 we introduce a viewdependent discretization algorithm. This algorithm can also work as a priority schema. In section 4 two methods are presented in order to obtain which pixel should be sampled next based on the quality measure for a pixel. In section 5 we present our conclusions and future work.

\section{Previous work}

In our previous work $[5,8,9,7]$, we introduced discrete and continuous information theory measures [1,2] of the visibility complexity in a scene. Next, we review the different definitions and results needed in this paper. 
Discrete mutual information in flatland is defined by

$$
I_{s}^{d}(S)=\sum_{i \in S} \sum_{j \in S} \ell_{i} F_{i j} \log \frac{F_{i j}}{\ell_{j}}
$$

where $S$ is the set of patches of the scene $\left(|S|=n_{p}\right), F_{i j}$ is the form factor between the patches $i$ and $j$, and $\ell_{i}=\frac{L_{i}}{L_{T}}$ is the relative length of patch $i$ ( $L_{i}$ is the length of patch $i$ and $L_{T}$ is the total length of the scene or the sum of segment lengths).

Continuous mutual information in flatland is defined by

$$
I_{s}^{c}=\iint_{x, y \in \mathcal{L}} \frac{F(x, y)}{L_{T}} \log \left(L_{T} F(x, y)\right) d L_{x} d L_{y}
$$

where $\mathcal{L}$ is the set of segments that forms the environment, $x$ and $y$ are points on segments of the environment and $F(x, y)$ is the differential form factor between $x$ and $y$. This integral can be solved by Monte Carlo integration [5] and the computation can be done efficiently by casting global lines uniformly distributed upon segments [10] (see figure 1). Thus, continuous mutual information can be approximated by

$$
\begin{aligned}
I_{s}^{c} & \simeq \frac{1}{N} \sum_{k=1}^{N} \log \left(L_{T} F\left(x_{k}, y_{k}\right)\right) \\
& =\frac{1}{N} \sum_{k=1}^{N} \log \left(\frac{L_{T} \cos \theta_{x_{k}} \cos \theta_{y_{k}}}{2 d\left(x_{k}, y_{k}\right)}\right)
\end{aligned}
$$

where $\theta_{x_{k}}$ and $\theta_{y_{k}}$ are the angles which the normals at $x_{k}$ and $y_{k}$ form with the segment joining $x_{k}$ and $y_{k}, d\left(x_{k}, y_{k}\right)$ is the distance between $x_{k}$ and $y_{k}, N$ is the total number of pairs of points considered, which is equal to the total number of intersections divided by two, and the value of $F(x, y)$ is $\frac{\cos \theta_{x} \cos \theta_{y}}{2 d(x, y)}$ for mutually visible points and zero otherwise.

Both measures, discrete and continuous mutual information, express the average information transfer in a scene. In $[5,8]$ continuous scene visibility mutual information has been proposed as an absolute measure of the complexity of scene visibility and discrete mutual information as a complexity measure of visibility of a discretized scene.

We define next discrete and continuous mutual information field at a point in flatland [7] which is related to the increase of complexity when an object is inserted at this point (see [9]). Note that we deal with the interior points of a $2 \mathrm{D}$ scene, which belong to the region strictly contained between the enclosure and the objects included in this one.

The contribution of patch $i \in S$ to the discrete mutual information field at point $x$ is

$$
I_{p}^{d}(x, i)=F_{p}(x, i) \log \left(\frac{F_{p}(x, i)}{\ell_{i}}\right)
$$

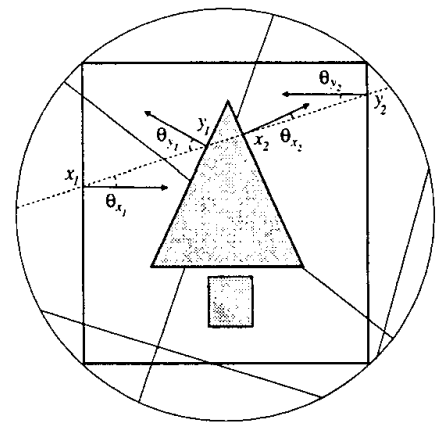

\section{Figure 1. Global lines are used to compute $I_{s}^{c}$. Lines are generated using a random point on a random diameter.}

where

$$
F_{p}(x, i)=\bar{\phi}(x, i)
$$

represents the fraction of random lines that exiting from point $x$ hit patch $i$ or the normalized visible angle $\bar{\phi}(x, i)$ subtended by patch $i[7]$. Thus, given a discretization $S$, the discrete mutual information field at point $x$ is given by

$$
I_{p}^{d}(x, S)=\sum_{i \in S} I_{p}^{d}(x, i)
$$

The contribution of patch $i \in S$ to the continuous mutual information field at point $x$ is

$$
I_{p}^{c}(x, i)=\int_{y \in \mathcal{L}_{i}} F_{p}(x, y) \log \left(L_{T} F_{p}(x, y)\right) d L_{y}
$$

where $F_{p}(x, y)$ represents the fraction of random lines that exiting from point $x$ hit differential $d L_{y}$ or the relative visible differential angle subtended by differential $d L_{y}$ [7].

$I_{p}^{c}(x, i)$ can be computed approximately by casting random lines from $x$ in all directions (similar to rays from a point light source, see figure 2):

$$
I_{p}^{c}(x, i) \simeq \frac{1}{N} \sum_{k=1}^{N(i)} \log \left(\frac{L_{T} \cos \theta_{y_{k}}}{2 \pi d\left(x, y_{k}\right)}\right)
$$

where $N$ is the total number of lines cast and $N(i)$ is the number of lines that hit patch $i$.

Thus, given any discretization $S$ of a scene, continuous visibility mutual information field at point $x$ is given by

$$
I_{p}^{c}(x)=\sum_{i \in S} I_{p}^{c}(x, i)
$$

Note that $I_{p}^{c}$ is independent of any discretization and can be computed by the above method:

$$
I_{p}^{c}(x) \simeq \frac{1}{N} \sum_{k=1}^{N} \log \left(\frac{L_{T} \cos \theta_{y_{k}}}{2 \pi d\left(x, y_{k}\right)}\right)
$$


where $N$ is the number of lines that hit the scene and $N=$ $\sum_{i \in S} N(i)$.

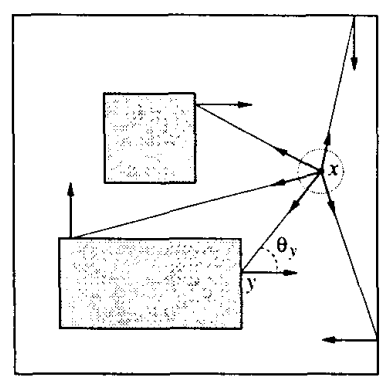

Figure 2. Random lines cast from a point $x$.

\section{View-dependent discretization}

In this section we present a proposition that allows us to derive a subdivision algorithm based on the mutual information field at a point $(6,9)$. This algorithm will be applied at different points of two different $2 \mathrm{D}$ scenes in order to show the feasibility of our approach.

\subsection{Continuous versus discrete mutual information at point $x$}

In [5] we showed that when a patch is refined into $n$ subpatches, discrete mutual information of a scene increases or remains the same, and continuous mutual information is the least upper bound to discrete mutual information: $I_{s}^{d} \leq I_{s}^{c}$. The optimal discretization corresponds with the one with minimal loss of information, or vice versa, the one with the highest mutual information.

Now, from the complexity measures at a point, we present a similar proposition:

Proposition 1 When a patch $i \in S$ is refined into $n$ subpatches, the discrete mutual information at point $x$ increases or remains the same.

This proof is shown in appendix A.

Corollary 1 For any discretization $S$ of a scene, the continuous mutual information at point $x$ is the least upper bound to discrete mutual information at point $x: I_{p}^{d}(x, S) \leq$ $I_{p}^{c}(x)$.

Proof: From the above proposition and as

$$
\lim _{|S| \rightarrow \infty} I_{p}^{d}(x, S)=I_{p}^{c}(x)
$$

we have that $I_{p}^{c}(x)$ is the least upper bound to $I_{p}^{d}(x, S)$.

\subsection{Subdivision algorithm}

From the results in the previous section, we propose a subdivision algorithm from a viewpoint (interior point of a scene).

As the difference $I_{p}^{c}(x)-I_{p}^{d}(x, S)$ represents the loss of information due to the discretization and our goal is to obtain the maximum information with the minimum number of patches, we choose to subdivide the patch $i$ that has the maximum loss of information, that is, the maximum potential information gain $\left(I_{p}^{c}(x, i)-I_{p}^{d}(x, i)\right)$.

We define the gradient over patch $i$ by

$$
\Delta(x, i)=I_{p}^{c}(x, i)-I_{p}^{d}(x, i)
$$

and the global gradient of the scene by

$$
\Delta(x, S)=\sum_{i \in S} \Delta(x, i)
$$

We also introduce the discretization accuracy as the quotient

$$
\delta(x, S)=\frac{I_{p}^{d}(x, S)}{I_{p}^{c}(x)}
$$

which takes values from 0 to 1 .

In the following subdivision algorithm the patches are successively divided into two equal parts. The discretization process will finish when a given discretization accuracy is reached. Also it could be possible to end the algorithm when a given number of patches is achieved.

1. Input set of scene patches $S$

2. Input point of view $x$

3. Input accuracy $\epsilon$

4. $I_{p}^{d}(x, S) \leftarrow 0$

5. $I_{p}^{c}(x) \leftarrow 0$

6. For each $i \in S$, do

(a) Compute $F_{p}(x, i)$ from (5)

(b) Compute $I_{p}^{d}(x, i)$ from (4)

(c) Compute $I_{p}^{c}(x, i)$ from (8)

(d) $\Delta(x, i) \leftarrow I_{p}^{c}(x, i)-I_{p}^{d}(x, i)$ from (11)

(e) $I_{p}^{d}(x, S) \leftarrow I_{p}^{d}(x, S)+I_{p}^{d}(x, i)$ from (6)

(f) $I_{p}^{c}(x) \leftarrow I_{p}^{c}(x)+I_{p}^{c}(x, i)$ from (9)

7. $\delta \leftarrow \frac{I_{p}^{d}(x, S)}{I_{p}^{c}(x)}$ from (13)

8. While $\delta<\epsilon$, do 

(a) $k \leftarrow$ Index of $\max _{i \in\{1 .|S|\}}(\Delta(x, i))$
(b) Subdivision of patch $k \in S$ into $\left\{k_{0}, k_{1}\right\}$
(c) $S \leftarrow(S-\{k\}) \cup\left\{k_{0}, k_{1}\right\}$
(d) Compute $\left(F_{p}\left(x, k_{0}\right), F_{p}\left(x, k_{1}\right)\right)$
(e) Compute $\left(I_{p}^{d}\left(x, k_{0}\right), I_{p}^{d}\left(x, k_{1}\right)\right)$
(f) Compute $\left(I_{p}^{c}\left(x, k_{0}\right), I_{p}^{c}\left(x, k_{1}\right)\right)$
(g) $I_{p}^{d}(x, S) \leftarrow I_{p}^{d}(x, S)-I_{p}^{d}(x, k)+$ $I_{p}^{d}\left(x, k_{0}\right)+I_{p}^{d}\left(x, k_{1}\right)$
(h) $\Delta\left(x, k_{0}\right) \leftarrow I_{p}^{c}\left(x, k_{0}\right)-I_{p}^{d}\left(x, k_{0}\right)$
(i) $\Delta\left(x, k_{1}\right) \leftarrow I_{p}^{c}\left(x, k_{1}\right)-I_{p}^{d}\left(x, k_{1}\right)$
(j) $\delta \leftarrow \frac{I_{p}^{d}(x, S)}{I_{p}^{c}(x)}$

9. Output new set of scene patches $S$

\subsection{Results}

The behaviour of this algorithm is shown in figures 3 , 4 and 5 , and table 1 . These figures represent different discretizations from different viewpoints. As we can observe, the discretization is finer in the discontinuity regions and also on the patches which are not well oriented with respect to the point. Over these patches we obtain (11) a higher gradient $\Delta$, basically due to the difference of distances between the viewpoint and the points on a patch.

\begin{tabular}{|c|c||c|c|c|c|}
\hline scene & lines cast & $n_{p}$ & $I_{p}^{d}$ & $I_{p}^{c}$ & $100 \delta$ \\
\hline \hline 3(a) & $10^{4}$ & 120 & 1.6105 & 1.6122 & 99.90 \\
\hline 3(b) & $10^{4}$ & 120 & 1.6266 & 1.6279 & 99.92 \\
\hline 3(c) & $10^{4}$ & 120 & 2.2987 & 2.2999 & 99.95 \\
\hline 4(a) & $10^{5}$ & 224 & 2.7548 & 2.7681 & 99.52 \\
\hline 4(b) & $10^{5}$ & 224 & 3.1847 & 3.1884 & 99.89 \\
\hline
\end{tabular}

Table 1. Final values obtained by the subdivision algorithm for the scenes of figures 3 and 4. $n_{p}$ is the final number of patches, $I_{p}^{d}$ and $I_{p}^{c}$ are the discrete and continuous mutual information field at a point respectively, and $100 \delta$ is the accuracy achieved.

In figure 5(a) and 5(b) we show the convergence of $I_{p}^{d}$ towards $I_{p}^{c}$ for the scenes of figures 3 and 4 respectively. Table 1 shows the values corresponding to the final discretization obtained. In figure 5 , the points marked represent the number of patches in which $\delta=0.99$ is obtained. Note that, in the examples presented, a good discretization is achieved first from the most complex viewpoints. To compute the subdivision of figures 3 and 4 we cast $10^{4}$ and $10^{5}$ random lines respectively.

\section{Pixel sampling}

In this section we define the quality of a pixel and give two heuristics based on this definition. These are applied to pixel sampling and compared with a heuristic based on depth difference [11].

\subsection{Pixel quality measures}

We consider here pixels defined on a circle instead of a line, although there is practically no difference in the final results. First we define continuous mutual information for a pixel.

Pixel continuous mutual information or pixel quality is defined by

$$
\begin{aligned}
& I_{p}^{c}\left(x, p_{i}\right) \\
= & I_{p}^{c}\left(x, S\left(x, p_{i}\right)\right) \\
= & \int_{y \in \mathcal{S}\left(x, p_{i}\right)} F_{p}(x, y) \log \left(L_{T} F_{p}(x, y)\right) d L_{y}
\end{aligned}
$$

where $p_{i}$ is a pixel and $S\left(x, p_{i}\right)$ is the set of patches visible through pixel $p_{i}$ from point $x$.

In a similar way we have shown in the previous work, pixel quality can be computed casting random lines from viewpoint $x$ to the scene through pixel $p_{i}$ :

$$
I_{p}^{c}\left(x, p_{i}\right) \simeq \frac{1}{N} \sum_{k=1}^{N\left(x, p_{i}\right)} \log \left(\frac{L_{T} \cos \theta_{y_{k}}}{2 \pi d\left(x, y_{k}\right)}\right)
$$

where $N$ is the total number of lines cast, $N\left(x, p_{i}\right)$ is the number of random lines through pixel $p_{i}$ from point $x$, and $N=\sum_{i} N\left(x, p_{i}\right)$.

Pixel line quality is given by

$$
I_{p}^{c}\left(x, p_{i}, k\right)=\log \left(\frac{L_{T} \cos \theta_{y_{k}}}{2 \pi d\left(x, y_{k}\right)}\right)
$$

where $k \in\left\{1 . . N\left(x, p_{i}\right)\right\}$, and represents the contribution of each line to the pixel quality.

\subsection{Sampling heuristics}

We present two different heuristics which will be evaluated in comparison to a depth heuristic defined in [11] as

$$
\Gamma\left(x, p_{i}\right)=1-\frac{D_{\min }}{D_{\max }}
$$

where $D_{\min }$ and $D_{\max }$ are respectively the minimum and maximum distances from viewpoint $x$ to the scene through pixel $p_{i}$.

This heuristic was used to drive a priority schema for pixel sampling purposes. It was the geometrical part of a combined color and depth difference measure. 


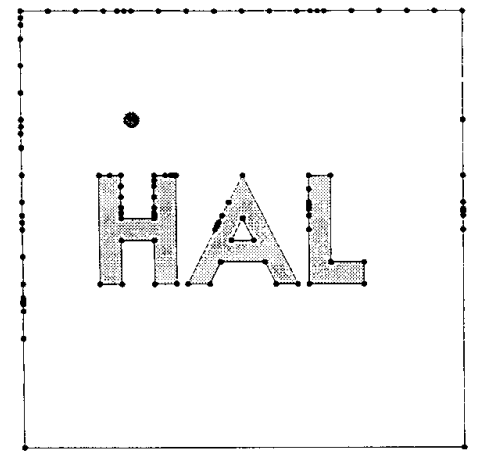

(a)

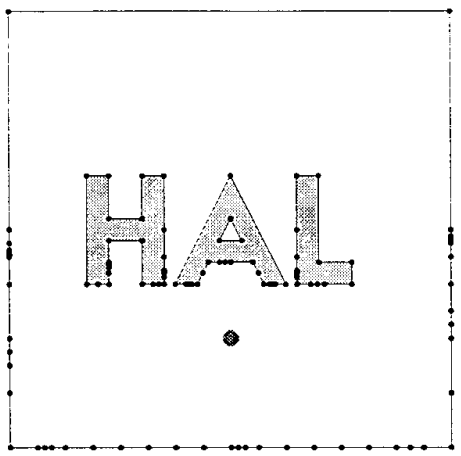

(b)

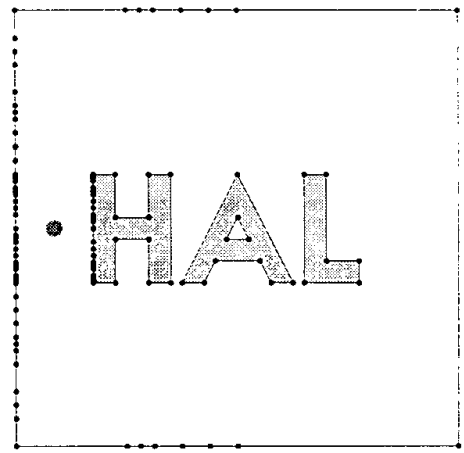

(c)

Figure 3. Three different discretizations of the same scene from different viewpoints (represented by a little circle) obtained by casting $10^{4}$ lines. The final values are shown in table 1.

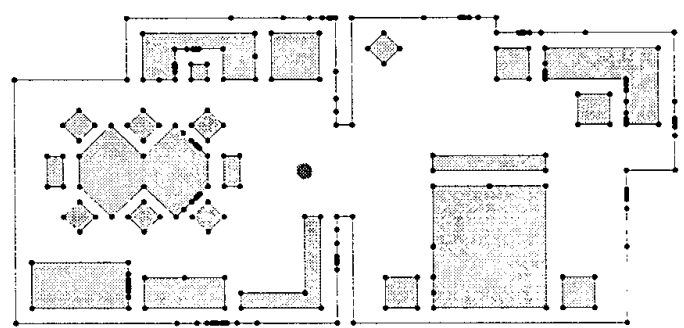

(a)

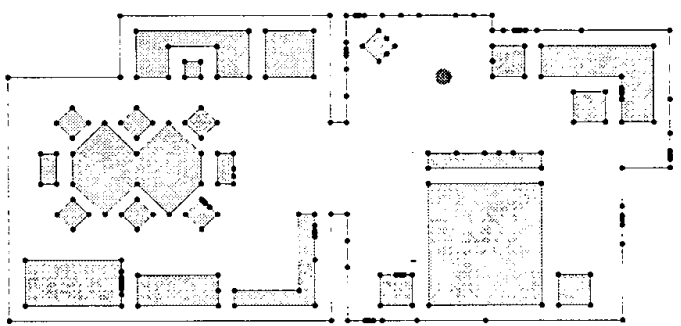

(b)

Figure 4. Two different discretizations of the same scene from different viewpoints (represented by a little circle) obtained by casting $10^{5}$ lines. The final values are shown in table 1.

Our first approach calculates for each pixel the sum of differences between successive pixel line qualities which can be expressed by

$$
\begin{aligned}
& \sum_{k=1}^{\Lambda\left(x, p_{i}\right)}\left|I_{p}^{c}\left(x, p_{i}, k\right)-I_{p}^{c}\left(x, p_{i}, k+1\right)\right| \\
= & \sum_{k=1}^{N\left(x, p_{i}\right)-1}\left|\log \left(\frac{L_{T} \cos \theta_{y_{k}}}{2 \pi d\left(x, y_{k}\right)}\right)-\log \left(\frac{L_{T} \cos \theta_{y_{k+1}}}{2 \pi d\left(x, y_{k+1}\right)}\right)\right| \\
= & \sum_{k=1}^{N\left(x, p_{i}\right)-1}\left|\log \left(\frac{d\left(x, y_{k+1}\right) \cos \theta_{y_{k}}}{d\left(x, y_{k}\right) \cos \theta_{y_{k+1}}}\right)\right|
\end{aligned}
$$

This measure can be interpreted as representing the orography of the region seen through the pixel and will be used to obtain the proportion of lines cast for each pixel.

The second approach takes only into account the difference between maximum and minimum line quality for each pixel and is expressed by

$$
\Upsilon\left(x, p_{i}\right)=\max _{k=1}^{N\left(x, p_{i}\right)}\left(I_{p}^{c}\left(x, p_{i}, k\right)\right)-\min _{k=1}^{N\left(x, p_{i}\right)}\left(I_{p}^{c}\left(x, p_{i}, k\right)\right.
$$

This is an analogous to the depth difference measure and can be interpreted as representing the gradient of the region seen through the pixel. As in the first approach, the number of lines cast for each pixel is proportional to this value.

While the distance heuristic only considers the distance between the viewpoint and the segment, in our approaches, pixel line complexity conveys two kinds of information: distance and orientation. So, our two heuristics discriminate discontinuities and also orientation changes (vertices). To see this, pixel line quality can be split in the following way

$$
\begin{aligned}
& I_{p}^{c}\left(x, p_{i}, k\right) \\
= & \log \left(\frac{L_{T} \cos \theta_{y_{k}}}{2 \pi d\left(x, y_{k}\right)}\right)
\end{aligned}
$$




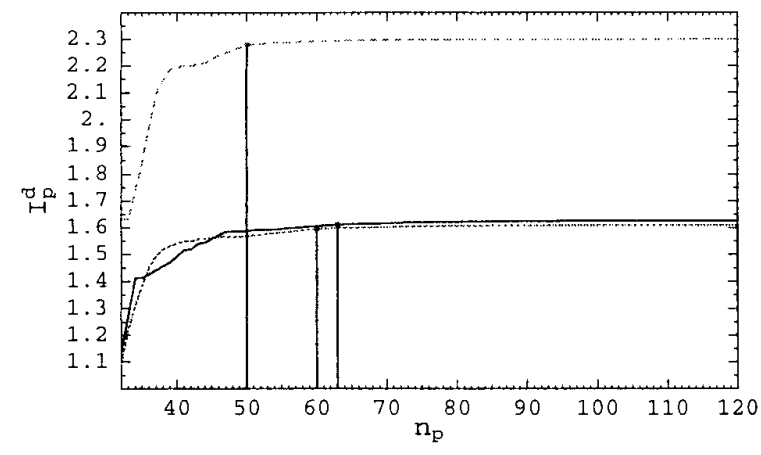

(a)

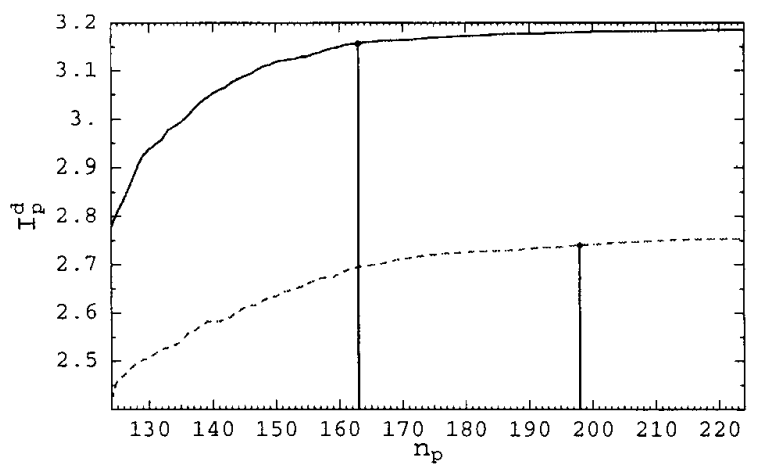

(b)

Figure 5. Plots (a) and (b) correspond to figures 3 and 4 respectively. In (a), dot, continuous, and dashed lines correspond to scenes $3(\mathbf{a}, \mathbf{b}, \mathbf{c})$, and an accuracy $\delta=0.99$ is achieved with $n_{p}=$ $(60,63,50)$ respectively. In (b), dashed, and continuous lines correspond to scenes $\mathbf{4}(\mathbf{a}, \mathbf{b})$, and the same accuracy is achieved with $n_{p}=(198,163)$ respectively.

$$
=\log \left(\cos \theta_{y_{k}}\right)+\log \left(\frac{L_{T}}{2 \pi d\left(x, y_{k}\right)}\right)
$$

where the first term in (20) represents the patch orientation contribution and the second represents the distance contribution. In this way our approach can be seen as the combination of the ones based on orientation and depth, respectively.

\subsection{Results}

In figures 6 and 7 we show the results of applying the above three heuristics. Figures (a), (b), and (c) are obtained respectively from the depth $(\Gamma)$, sum of differences $(\Lambda)$, and gradient $(\Upsilon$ ) heuristics.

In the images that correspond to the depth heuristic (figures 6(a) and 7(a)) we observe that some edges remain unsampled as, contrary to ours, the depth heuristic does not capture a change in orientation from edge to edge. Compare for instance the corners in the bedroom in figures 7(ii). In general our heuristics behave better by obtaining a finer sampling where it is more needed. On the other hand a possible drawback in our approaches can appear when the distance factor is balanced with the orientation factor. This happens for instance at the $\mathbf{L}$ corner in figures 6(i). We are currently considering a solution to this problem by taking separately the two components of the measure (20).

\section{Conclusions and future work}

We have presented in this paper several view-dependent information theory based quality measures for pixel sampling and scene discretization in flatland. The measures are based on the definition for the mutual information of a line. Results show that they are very promising and compare well with depth measures. Although this is a purely geometrical approach we plan in our future work to extend it to deal also with color. The extension to 3D scenes, although not yet implemented, is straightforward.

\section{Acknowledgements}

This project has been funded in part with grant numbers TIC-98-586-C03 and TIC-98-973-C03 of the Spanish Government and with a Spanish-Austrian joint action number HU-1998-0015 of the Ministerio de Educación y Cultura.

\section{A Proof of the proposition}

Let us imagine a point $x$ in a discretized scene where $|S|=n_{p}$ and discrete mutual information field at point $x$ is $I_{p}^{d}(x, S)$. If any patch $i$ is divided into $n>0$ subpatches $S_{i}=\left\{i_{k} \mid k \in\{1 . . n\}\right\}$, we have to show that discrete mutual information at point $x$ of the new discretization $S^{\prime}=(S-$ $\{i\}) \cup S_{i}$ (where $\left|S^{\prime}\right|=n_{p}+n-1$ ) fulfills $I_{p}^{d}(x, S) \leq$ $I_{p}^{d}\left(x, S^{\prime}\right)$.

First, we show that $I_{p}^{d}(x, i) \leq I_{p}^{d}\left(x, S_{i}\right)$. From the following equalities $F_{p}(x, i)=\sum_{k=1}^{n} F_{p}\left(x, i_{k}\right)$ and $\ell_{i}=$ $\sum_{k=1}^{n} \ell_{i_{k}}$ we obtain

$$
\begin{aligned}
& I_{p}^{d}(x, i) \leq I_{p}^{d}\left(x, S_{i}\right) \\
\equiv & F_{p}(x, i) \log \left(\frac{F_{p}(x, i)}{\ell_{i}}\right) \leq \sum_{k=1}^{n} I_{p}^{d}\left(x, i_{k}\right)
\end{aligned}
$$




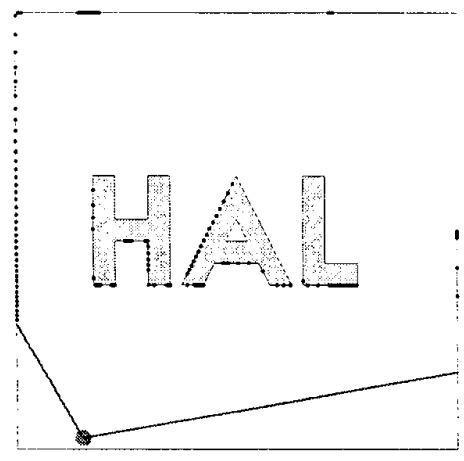

(a.i)

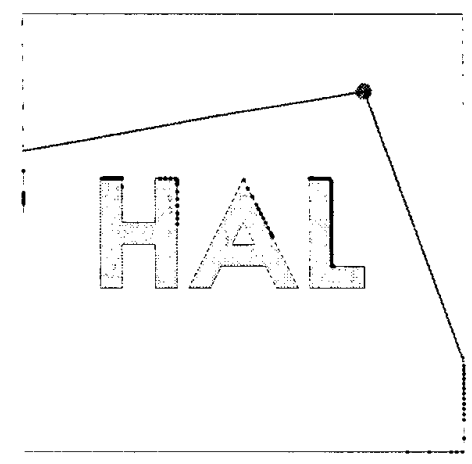

(a.ii)

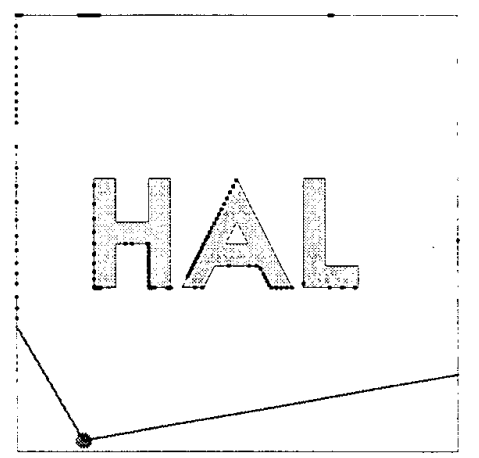

(b.i)

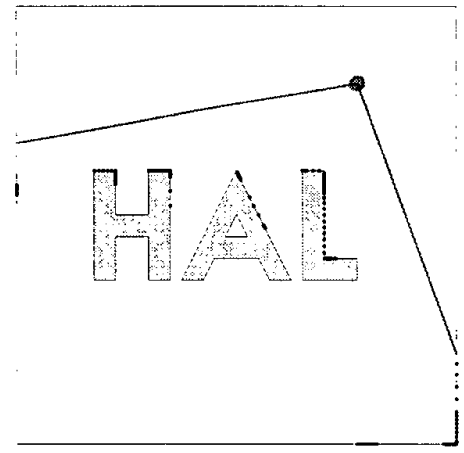

(b.ii)

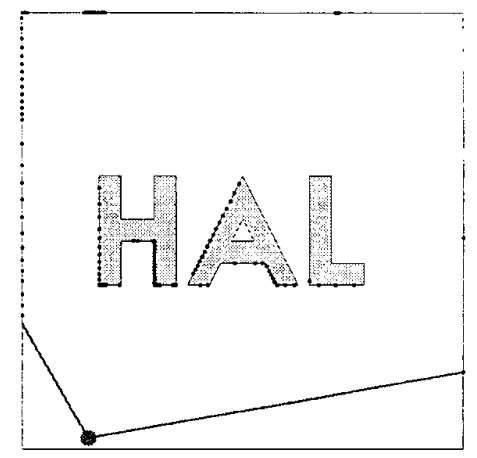

(c.i)

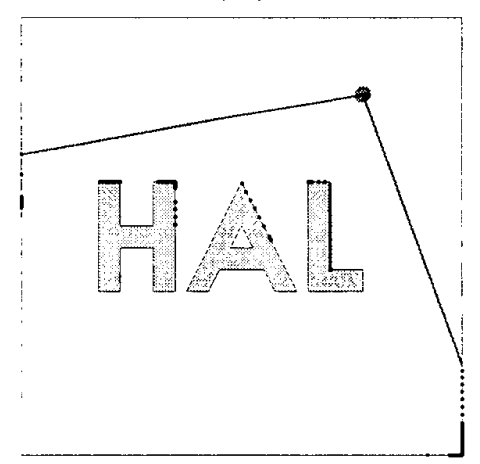

(c.ii)

Figure 6. The same scene with six different pixel samplings. Viewpoint is represented by a little circle. Field of view has $110^{\circ}$ and $100^{\circ}$ for figures (i) and (ii) respectively, 150 lines have been cast and 30 pixels are used. Figures (a), (b), and (c) correspond to $\Gamma, \Lambda$, and $\Upsilon$ heuristics respectively.

$$
\begin{aligned}
& \equiv \quad\left(\sum_{k=1}^{n} F_{p}\left(x, i_{k}\right)\right) \log \left(\frac{\sum_{k=1}^{n} F_{p}\left(x, i_{k}\right)}{\sum_{k=1}^{n} \ell_{i_{k}}}\right) \\
& \leq \sum_{k=1}^{n} F_{p}\left(x, i_{k}\right) \log \left(\frac{F_{p}\left(x, i_{k}\right)}{\ell_{i_{k}}}\right) \\
& \equiv \text { True }
\end{aligned}
$$

The last step is true for the concavity of the logarithm function for non-negative numbers:

$$
\left(\sum_{k=1}^{n} a_{k}\right) \log \left(\frac{\sum_{k=1}^{n} a_{k}}{\sum_{k=1}^{n} b_{k}}\right) \leq \sum_{k=1}^{n} a_{k} \log \left(\frac{a_{k}}{b_{k}}\right)
$$

where $\left(a_{k}, b_{k}\right)=\left(F_{p}\left(x, i_{k}\right), \ell_{i_{k}}\right)$.

Now we have

$$
\begin{aligned}
& I_{p}^{d}(x, S) \leq I_{p}^{d}\left(x, S^{\prime}\right) \\
\equiv & \sum_{k \in S} I_{p}^{d}(x, k) \leq \sum_{k \in S^{\prime}} I_{p}^{d}(x, k) \\
\equiv & \sum_{k \in(S-\{i\}) \cup\{i\}} I_{p}^{d}(x, k) \leq \sum_{k \in(S-\{i\}) \cup S_{i}} I_{p}^{d}(x, k)
\end{aligned}
$$

$$
\begin{aligned}
& \equiv\left(\sum_{k \in S-\{i\}} I_{p}^{d}(x, k)\right)+I_{p}^{d}(x, i) \\
& \quad \leq\left(\sum_{k \in S-\{i\}} I_{p}^{d}(x, k)\right)+\left(\sum_{k \in S_{i}} I_{p}^{d}(x, k)\right) \\
& \equiv I_{p}^{d}(x, i) \leq I_{p}^{d}\left(x, S_{i}\right) \\
& \text { 曰 } \operatorname{True}
\end{aligned}
$$

\section{References}

[1] R. Blahut. Principles and Practice of Information Theory. Addison-Wesley, 1987.

[2] T. Cover and J. Thomas. Elements of Information Theory. Wiley Series in Telecommunications, 1991.

[3] L. Darsa and B. C. Silva. Multi-resolution representation and reconstruction of adaptively sampled images. In Proceedings of IX Brazilian Symposium on Computer Graphics and Image Processing (SIBGRAPI'96), pages 321-328, October 1996.

[4] L. Darsa, B. C. Silva, and A. Varshney. Navigating static environments using image-space simplification and morphing. 


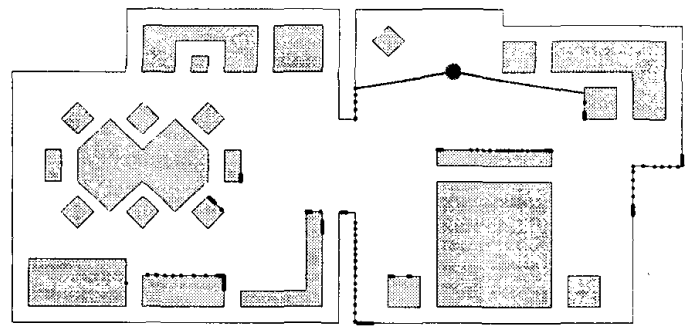

(a.i)

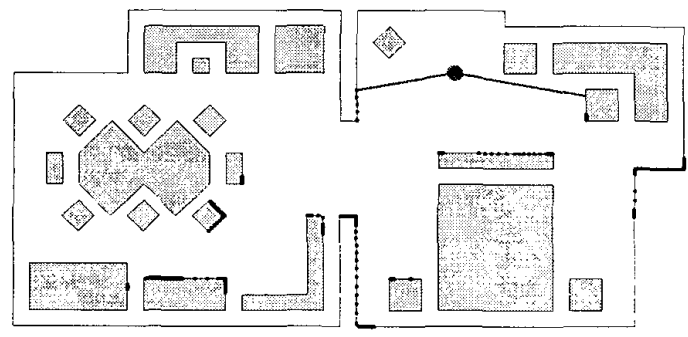

(b.i)

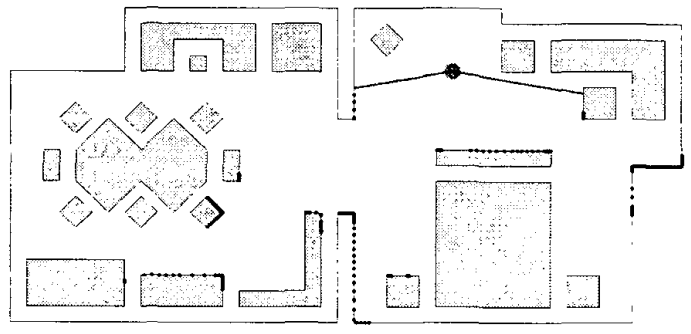

(c.i)

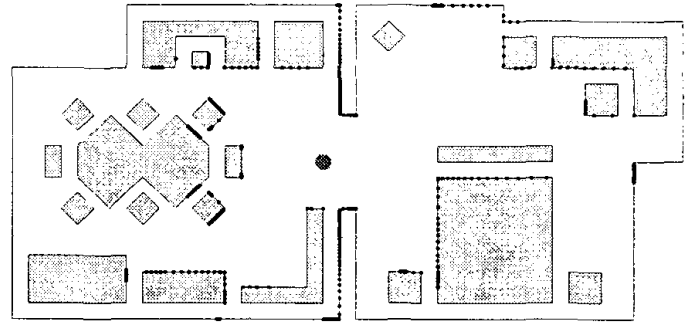

(a.ii)

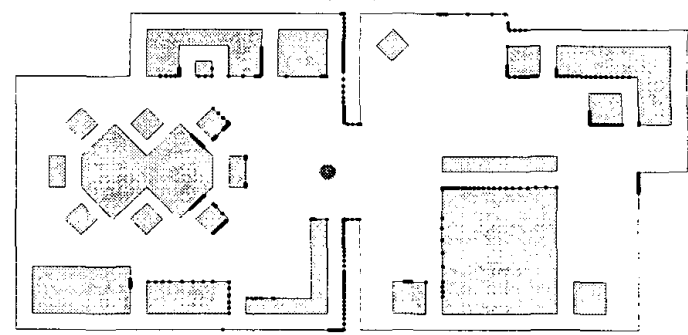

(b.ii)

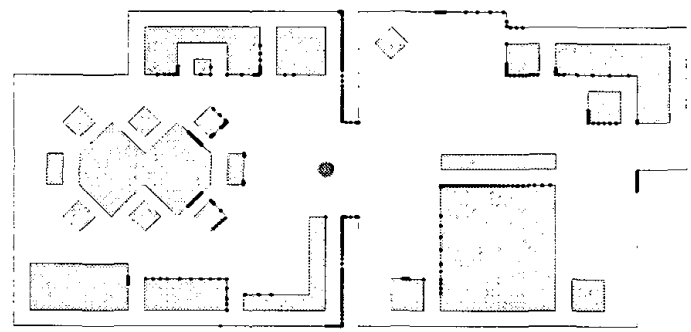

(c.ii)

Figure 7. The same scene with six pixel samplings. Viewpoint is represented by a little circle. For figures (i) the field of view has $190^{\circ}, 200$ lines have been cast, and 40 pixels are used, and for figures (ii) the field of view has $360^{\circ}, 400$ lines have been cast, and 80 pixels are used. Figures (a), (b), and (c) correspond to $\Gamma, \Lambda$, and $\Upsilon$ heuristics respectively.

1997 Symposium on Interactive 3D Graphics, pages 25-34, April 1997.

[5] M. Feixas, E. del Acebo, P. Bekaert, and M. Sbert. An information theory framework for the analysis of scene complexity. Computer Graphics Forum (Proceedings of Eurographics'99), 18(3):95-106, September 1999.

[6] K. Pulli, M. F. Cohen, T. Duchamp, H. Hoppe, L. Shapiro, and W. Stuetzle. View-based rendering: Visualizing real objects from scanned range and color data. In J. Dorsey and P. Slusallek, editors, Rendering Techniques' 97 (Proceedings of the 8th Eurographics Workshop on Rendering), pages 2334, Vienna, Austria, June 1997. Springer-Verlag Wien-New York. Held in St. Etienne, France.

[7] J. Rigau, M. Feixas, and M. Sbert. Information theory point measures in a scene. Research Report IIiA-00-08-RR, Institut d'Informàtica i Aplicacions, Universitat de Girona, Girona, Spain, 2000.
[8] J. Rigau, M. Feixas, and M. Sbert. Scene visibility complexity in flatland. Research Report IliA-00-03-RR, Institut d'Informàtica i Aplicacions, Universitat de Girona, Girona, Spain, 2000.

[9] J. Rigau, M. Feixas, and M. Sbert. Visibility complexity of a region in flatland. In Short Presentations of Eurographics'00, pages 159-163, Interlaken, Switzerland, August 2000.

[10] M. Sbert. The Use of Global Random Directions to Compute Radiosity. Global Monte Carlo Methods. PhD thesis, Universitat Politècnica de Catalunya, Barcelona, Spain, November 1996.

[11] M. Simmons and C. H. Séquin. Tapestry: A dynamic mesh-based display representation for interactive rendering. In B. Péroche and H. Rushmeier, editors, Rendering Techniques 2000 (Proceedings of the 1lth Eurographics Workshop on Rendering), pages 329-340, Vienna, Austria, June 2000. Springer-Verlag Wien-New York. 